\title{
BJMHR
}

British Journal of Medical and Health Research

Journal home page: www.bjmhr.com

\section{Chronotherapeutics - A Novel Drug Delivery Systems In The Treatment of Circadian Rhythms Diseases}

\section{Murali Krishna Goud* A. Mallishwar, A. Manoj Kumar, A. Trinath, B.Swapna 1.Pulla Reddy Institute of Pharmacy, Domadugu (V), Gummadidala (M), SangaReddy- 502313}

\section{ABSTRACT}

Chronotherapeutics also known as pulsatile drug delivery system deals with the study of the temporal changes in absorption, distribution, metabolism and elimination and thus takes into account the influence of time of administration on these different steps and it focuses on the release of a drug after a lag time at a particular site in order to maintain constant blood levels of a particular drug matching circadian rhythms of various diseases. Circadian time dependent differences are also seen in pharmacokinetics of many classes of medications like cardiovascular active drugs, NSAID's, antidepressants, anti hypertensives, local anesthetics, $\mathrm{H}_{1}$ and $\mathrm{H}_{2}$ antagonists etc. The role of circadian rhythms in the mechanisms of disease and the pharmacokinetics and pharmacodynamics of medications constitutes a challenge to drugdiscovery and drug-delivery scientists. We must strive to develop intelligent drug-delivery systems that can affect a target cell or organ system at that circadian time when it is possible to optimize desired therapeutic outcomes and minimize or avert adverse effects. The recent advances in pulsatile drug delivery technology are CODAS, ACCU-BREAK, SODAS, IPDAS, DMDS Technology etc.

Keywords: Chronotherapeutics, Drug delivery, pulsatile drug delivery systems , Drug administration routes, CODAS, ACCU-BREAK. 


\section{INTRODUCTION $^{[1]}$}

The goal in drug delivery research is to meet therapeutic needs relating to particular pathological conditions by developing new formulations. Research in the chronopharmacological field has demonstrated the importance of biological rhythms in drug therapy, and this has brought a new approach to the development of oral drug delivery systems. Different technologies are being utilized in the development of triggered, pulsatile, controlled and programmed drug delivery devices has intensified in recent years. Chronotherapeutics is the discipline concerned with the delivery of drugs according to the intrinsic activities of a disease over a certain period of time because the biochemical, physiological and pathological variations over a $24 \mathrm{~h}$ period in humans (Figure 1) have been occurred. Chronotherapeutics deals with the medical treatment according to the human daily working cycle that corresponds to a person's daily, monthly, seasonal or yearly biological clock or in order to maximize the health benefits and minimize the adverse effects. The main goal of chronotherapeutics is to match the timing of treatment with the intrinsic timing of illness. Optimum therapy is given when the right amount of drug is delivered to the correct target organ at the most appropriate time. If symptoms of a disease are varied the circadian rhythms also varied the drug release. In the treatment of many diseases chronotherapeutics drug delivery offers a new approach in the pharmacologic interventions design for the effective treatment in the different types of diseases.

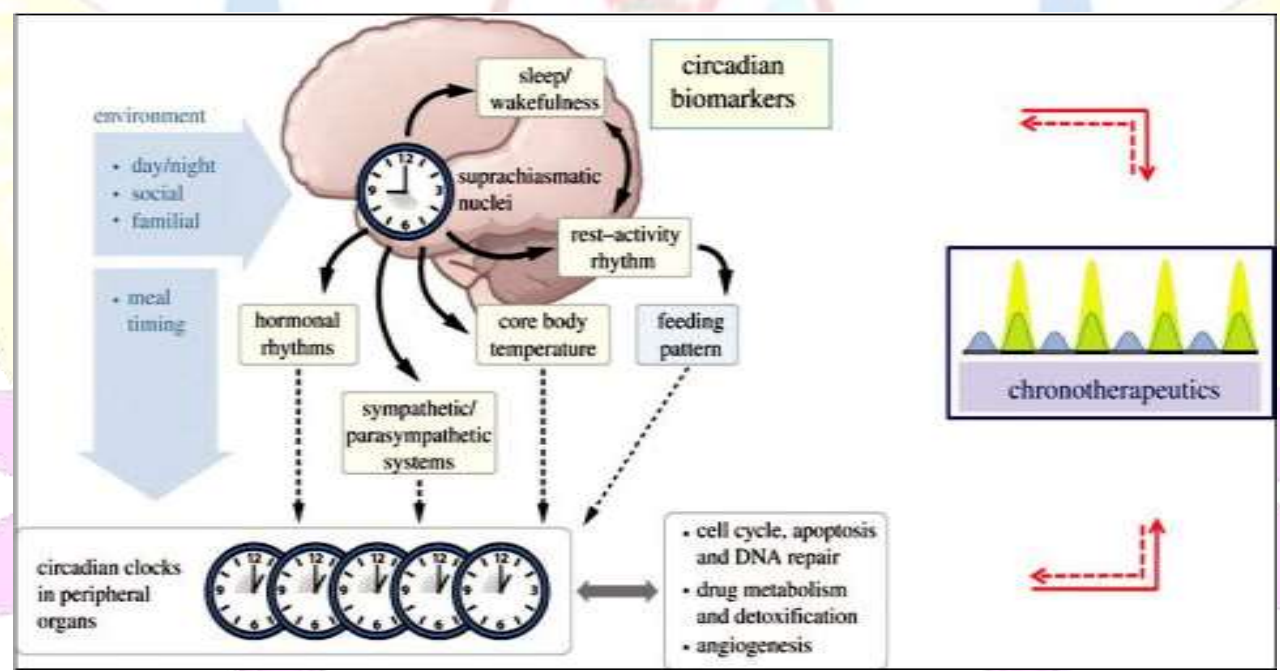

Figure 1: Chronotheraputics - circadian cycle of human body ${ }^{[1]}$

The "chronotherapeutics" term is mainly new in the field of drug delivery and in the treatment method. It is defined as the widespread term in which disease follow the circadian rhythm which undergoes the metabolic changes. Chronotherapeutics is defined as the method in which drug availability is matched with the rhythms of the disease according to the time structure which results in the maximum therapeutic effects and less adverse effects. 


\section{Biological rhythms ${ }^{[2,3]}$}

A biological rhythm is a self-sustaining oscillation of endogenous origin. The spectrum of biological rhythms is broad as displayed in Table 1. Short-period rhythms of a second or so are quite common; the high frequency oscillations in the electrical impulses of the central and autonomic nervous systems and the high frequency pulsatile secretions of the neuro endocrine system are but a few examples. Intermediate-period rhythms show oscillations as short as a few hours to as long as 6 days. Included in this category are the ultradian $(<20$ h), light intensities are thought to be the major environmental cue involved in circadian entrainment. Light- signals are perceived by photoreceptor cells in the retina and transmitted to neurons of the SCN via the retino hypothalamic tract. A great deal of research shows that the inherited period of the human pacemaker clock is not precisely $24 \mathrm{~h}$. In fact, in most people, it is somewhat longer, closer to $25 \mathrm{~h}$. Environmental times, termed synchronizers or zeitgebers, the strongest one being the daily light-dark cycle occurring in conjunction with the wake-sleep routine, set the inherited pacemaker circadian timekeeping systems to $24 \mathrm{~h}$ each day. The human circadian time structure was depicted in Table 1.

Table 1: Spectrum of biological rhythms ${ }^{[2,3]}$

\begin{tabular}{lll}
\hline Period $(\tau)$ & Major rhythmic components \\
Short $[\tau<0.5 \mathrm{~h}]$ & Pulsatiles & $(0.1 \mathrm{~s}<\tau<1 \mathrm{~s})$ \\
Intermediate & Circadian & $(20 \mathrm{~h}<\tau<28 \mathrm{~h})$ \\
& Ultradian & $(0.5 \mathrm{~h}<\tau<20 \mathrm{~h})$ \\
{$[0.5 \mathrm{~h}<\tau<6$ days $]$} & Infradian $\quad(28 \mathrm{~h}<\tau<6$ days $)$ \\
Long Period & $\begin{array}{l}\text { Circamensual }(\tau \sim 30 \text { days }) \\
\text { Circaseptan }\end{array}$ & $(\tau \sim 7$ days $)$ \\
& Circannual & $(\tau \sim 1$ year $)$ \\
\hline
\end{tabular}

\section{Circadian time structure ${ }^{[2,3]}$}

The results of numerous biological rhythm studies help define the temporal organization of human beings. One means of illustrating the human circadian time structure is to depict the peak time of 24-h rhythms on a clock-like diagram like that shown in Figure 2. This figure shows the peak time of a select number of human circadian rhythms in relation to the typical synchronizer routine of most human beings-sleep in darkness from 10.30 P.M to 6.30 A.M and activity during the light of the day between 6.30 A.M and 10.30 P.M.

The peak gastric acid secretion, white blood cell count (WBC), calcitonin gene-related protein, and atrial natriuretic peptide occurs late at night or early in sleep. Growth and thyroid stimulating hormone (TSH), blood lymphocyte and eosinophil number, and plasma melatonin and prolactin crest during sleep as do the adreno corticotropic (ACTH), follicle stimulating (FSH), and luteinizing (LH) hormones. Plasma cortisol, renin activity, angiotensin, and 
aldosterone peak in the morning as do arterial compliance, vascular resistance, platelet aggregation, and blood viscosity. Hemoglobin and insulin concentrations peak at noon and in the afternoon, as do the spirometric measures of airways caliber $\mathrm{FEV}_{1}$ (forced expiratory volume in $1 \mathrm{~s}$ ) and PEF (peak expiratory flow rate).

The circadian rhythms of serum cholesterol and triglycerides and urinary diuresis crest early in the evening. The information conveyed in this figure clearly illustrates that the biochemistry and physiology of human beings are not constant; rather, they are variable in a predictable and coordinated manner during the $24 \mathrm{~h}$.

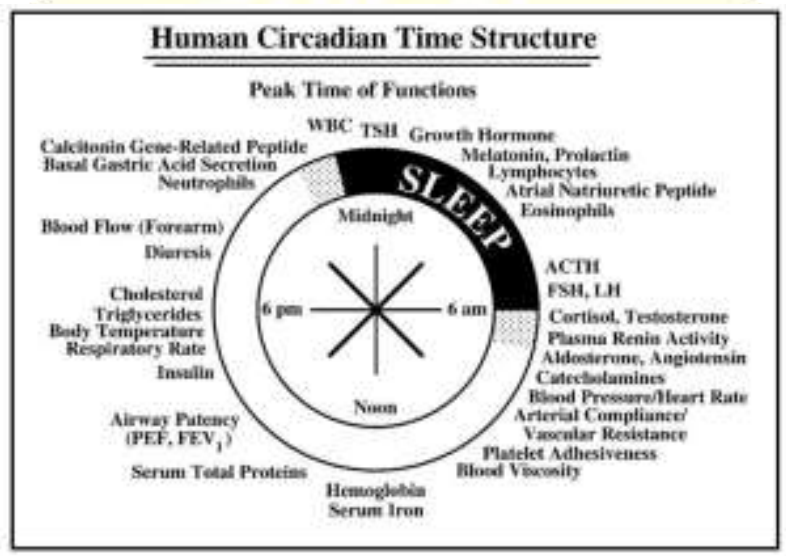

Figure 2: Human circadian time structure. Shown is the approximate peak time of circadian (24-h) rhythms of selected biological variables in persons adhering to a normal routine of daytime activity $(\sim 6-7$ a.m. to $\sim 10-11$ p.m.) alternating with nighttime sleep $^{[2,3]}$.

Chronotherapy ${ }^{[2,3]}$

Coordinating biological rhythms with medical treatment is known as chronotherapy, which allows for appropriate dosing of actives at the most suitable times of the day, thus improving efficacy and reducing undesirable side effects.

Advantages of Chronotherapy ${ }^{[2,3]}$

1. Chronotherapy is drug-free

2. Chronotherapy is more effective when a person sleeps for several hours.

3. While Chronotherapy patients often fall asleep this improves their condition and confidence as well.

4. Chronotherapy is different from other treatments because it got the beginning, middle, and an end. So one can predict easily the point at which it will work.

5. It gives you a new schedule like getting up and sleeping early which will be quite unusual for some days but it will give $\mathrm{u}$ a period to adjust psychologically

6. Improved stability

7. No risk of dose dumping 


\section{Disadvantages of Chronotherapy ${ }^{[2,3]}$}

1. It develops a non 24 hours sleep wake syndrome after the treatment as the person sleeps or over 24 hours during the treatment.

2. Person become less productive during chronotherapy and staying awake till the other schedule will be bit uncomfortable.

3. Medical supervision is mandatory for this therapy.

4. Large number of process variables.

5. Trained /skilled person is needed for manufacturing.

\section{CIRCADIAN RHYTHMS IN OCCURRENCE AND SEVERITY OF DISEASE ${ }^{[2,3]}$}

\section{Bronchial Asthma:}

Airway resistance, broncho constriction and exacerbation of symptoms increase progressively at night in asthmatic patients. Risk of asthma attack is 100 -fold greater during night time sleep than during daytime activity. Chronotherapy for asthma is aimed at getting maximal effect from bronchodilator medications during early morning hours. Daily or alternate day, morning dose of glucocorticoid medications such as methyl predisolone (Medrol) significantly moderates side effects and enhance therapeutic benefits. Oral predisolone administered at $3 \mathrm{pm}$ rather than at $8 \mathrm{am}$ has been shown to be highly effective in the treatment of nocturnal asthma. Evening once daily dosing of controlled release theophylline tablets (Uniphyl $400 \mathrm{mg}$ tablets) showed chronotherapeutical potential in the treatment of nocturnal and early-morning asthma. Many circadian dependent factors appear to contribute to the worsening of nocturnal asthmatic symptoms. For example, cortisol (an antiinflammatory substance) levels were highest at the time of awakening and lowest in the middle of the night, and histamine (a mediator of broncho constriction) concentrations peaked at a level that coincided with the greatest degree of broncho constriction at 4:00 am.

It is a good target for chronotherapy because bronco constriction and exacerbation of symptoms vary on circadian fashion. The enhanced understanding of the chronobiological impact upon the pathology of asthma, and the pharmacology and pharmacokinetics of the drugs used in its management, have led to new approaches to disease management and enhanced patient care.

\section{Pain:}

Pain threshold does not follow the same pattern in all tissues. The sensitivity threshold of the gingival to a cold stimulus was maximal at 18:00 $\mathrm{h}$ and reached a peak at 03:00 $\mathrm{h}(35 \%$ difference). Tooth sensitivity was lowest between 15:00 and 18:00 h, with a peak in pain intensity at 08:00 h (160\% increase). Circadian rhythms in acute pain have been also recorded, such as in dental surgery, with a morning peak during the first postoperative day. 
The peak of morphine use occurred at 09:00 $\mathrm{h}$ and was the least at 15:00 $\mathrm{h}$ in patients undergoing elective surgery.

The peak demand for morphine or hydromorphone occurred in the early morning and was lowest during the night in postoperative gynecologic patients.

\section{Arthritis:}

Patients with osteoarthritis tend to have less pain in the morning and more at night; while those with rheumatoid arthritis, have pain that usually peaks in the morning and decreases throughout the day. Chronotherapy for all forms of arthritis using NSAID's such as ibuprofen should be timed to ensure that the highest blood levels of the drug coincide with peak pain.

For osteoarthritis sufferers, the optimal time for a non steroidal anti-inflammatory drug such as Ibuprofen would be around noon or mid-afternoon.

The same drug would be more effective for people with rheumatoid arthritis when taken after the evening meal. There is a circadian rhythm in the plasma concentration of $\mathrm{C}$ - reactive protein and interleukin-6 of patients with rheumatoid arthritis. Symptoms of rheumatoid arthritis are most intense when awaking from night time sleep, while those of osteoarthritis are worse in the evening or at night. Chronopharmacological studies of once daily sustained release indomethacin preparation for the treatment of osteoarthritis have indicated that time of dosing influences tolerances and effectiveness

\section{Cardiovascular diseases:}

Several functions (e.g. BP, heart rate, stroke volume, cardiac output, blood flow) of the cardiovascular system are subject to circadian rhythms. For instance, capillary resistance and vascular reactivity are higher in the morning and decrease later in the day. Platelet aggregability is increased and fibrinolytic activity is decreased in the morning, leading to a state of relative hyper coagulability of the blood. It was postulated that modification of these circadian triggers by pharmacologic agents may lead to the prevention of adverse cardiac events. Cardiac events also occur with a circadian pattern. Numerous studies have shown an increase in the incidence of early-morning myocardial infarction, sudden cardiac death, stroke, and episodes of ischemia6. BP is at its lowest during the sleep cycle and rises steeply during the early morning awakening period. Most patients with essential hypertension have a similar circadian rhythm of BP as do normo tensive persons, although hypertensive patients have an upward shift in the profile. They have quite a marked rise in blood pressure upon awakening - called 'the morning surge'- that increase can be $3 \mathrm{~mm} \mathrm{Hg} / \mathrm{hour}$ (systolic) and $2 \mathrm{~mm} \mathrm{Hg} /$ hour (diastolic) for the first four to six hours after waking up. This is due to high catecholamine concentration in the early morning.

\section{Hypercholesterolemia:}


A circadian rhythm occurs during hepatic cholesterol synthesis. However, this rhythm varies according to individuals. Indeed, there is a large variation in plasma mevalonate concentrations between individuals. Therefore cholesterol synthesis is generally higher during the night than during daylight, and diurnal synthesis may represent up to $30 \%-40 \%$ of daily cholesterol synthesis. Many individuals display a paradoxical synthesis, with an inverted diurnal cholesterol synthesis. It seems therefore that cholesterol is synthesized during the night as well as during daylight; however the maximal production occurs early in the morning, i.e. $12 \mathrm{~h}$ after the last meal. Studies with HMG CoA reductase inhibitors have suggested that evening dosing was more effective than morning dosing. that cholesterol synthesis increases during the night. Free cholesterol levels are reported to be lowest at $2 \mathrm{pm}$ to $6 \mathrm{pm}$ and peak at $6 \mathrm{am}$. Chronotherapy can be achieved by timing the medication in accordance with circadian rhythm for hypercholesterolemia. Morning versus evening administration of $\mathrm{HMG}-\mathrm{CoA}$ - reductase antagonists.

\section{Diabetes:}

The circadian variations of glucose and insulin in diabetes have been extensively studied and their clinical importance in case of insulin substitution in type 1 diabetes has been previously discussed. The goal of insulin therapy is to mimic the normal physiologic pattern of endogenous insulin secretion in healthy individuals, with continuous basal secretion as well as meal-stimulated secretion. Providing basal insulin exogenously to patients with diabetes inhibits hepatic glucose production. Exogenous administration of mealtime doses promotes peripheral glucose uptake (i.e. it prevents postprandial increases in blood glucose concentration) as well as reducing hepatic glucose release.

\section{Epileptic seizure:}

Chronophysiology investigations considered at a rhythmometric level of resolution suggested several heuristic perspectives regarding (1) the central pathophysiology of epilepsy and (2) the behavioral classification of convulsive events..Such circulation studies also show that chronobiology rises some working hypotheses in psycho physiology and permits the development of new theoretical concepts in the field of neurological sciences. It is also well known that the brain area with the highest concentration in noradrenergic nerve terminals and non adrenergic nerve terminals and nor adrenaline (NA) have a circadian rhythm in their content of NA.

\section{Cerebrovascular accidents:}

The cerebrovascular accidents have been shown to occur on the first hours of morning between 10 am and 12 noons, and the incidence declines steadily during the evening and the midnight. A major objective of chronotherapy for cardiovascular disease is to deliver the drug 
in higher concentration during time of greatest need and in lesser concentrations when the need is less. ACE inhibitors are more effective when administered during night. Atenolol, Nifedipine and Amolodipine are more effective when administered at night.

The first chronotherapeutic therapy for hypertension and angina pectoris has recently been developed which matches drug delivery to the circadian pattern of blood pressure and rhythm of myocardial ischemia. Verapamil has been employed in this system where release is observed after 4-5 hours and continues for 18hours.

\section{Myocardial Infarction:}

Onset of myocardial infarction has been shown to be more frequent in the morning with $34 \%$ events occurring between 6 am and noon. Acute cardiac arrest and transient myocardial ischemia shows an increased frequency in morning. The causes for these findings have been suggested to be release of catecholamines, cortisol, increase in the platelet aggregation and vascular tone.

\section{Peptic Ulcer Disease:}

A histamine antagonist when given at night shows the better result unlike when given at regular intervals around the clock. This is because the more acid secretion, more pain and perforation of gastric and duodenal ulcers are more subjective at night rather than in day time. It is well established that patients with peptic ulcer disease often experience the greatest degree of pain near the time that they go to bed, as the rate of stomach acid secretion is highest at night. The timing of administration of ulcer medications has a significant impact on their therapeutic effect.

It is well established that the patients with better the peptic ulcer disease are often experiences the greatest degree of pain in the near time that the patient go to bed . The timing of administration of ulcer medications has a significant result on their therapeutic effect shows the best chronotherapeutics drug delivery in the drug administration in the treatment of ulcer treatment which shows the right treatment a significant impact upon treatment and this will lead the according to the rhythms and biological time structure.

\section{Parkinson's disease:}

Autonomic dysfunction seen in Parkinson's disease discloses many alterations in circadian rhythm of blood pressure, amplified diurnal blood pressure variability and postprandial hypotension But existence of circadian rhythm in this disease has not been evaluated. Clinical data shows daily fluctuations of motor activity pattern but the effect of the phase of the disease and the subsequent roles of drugs are difficult to estimate.

\section{Allergic rhinitis:}

Common symptoms of allergic rhinitis are sneezing, nasal rhinorrhea, red itchy eyes, nasal 
pruritus and nasal congestion .Each of the symptoms was found to occur most frequently before breakfast and in the morning and least frequently in the middle of the day. There are two phases of occurrence of allergic rhinitis i.e. early phase (developing within minutes) and late phase (manifesting after12-16 h).

The early phase happens due to release of histamine, prostaglandins, cytokines, TNF- $\alpha$, chemotactic factors etc resulting in sneezing, nasal itch, rhinorrhea. On the other hand late phase is shown due to elaboration, adhesion and infiltration of circulating leukocytes, $\mathrm{T}$ cells and eosinophils evoking nasal congestion, obstruction due to the exacerbation of inflammation of the nasal, sinus and other tissue of the upper airway.

\section{Sleep disorder:}

Many biological signalings e.g. sleep disorder occurring in the central and autonomous nervous systems show complex time structure with rhythm and pulsatile variations in multiple frequencies.

The time of sleep required by each person is usually constant, although there is a wide variation among individuals. Sleep consists of a rhythmic (circadian) combination of the changes in physiological, biochemical and psychological processes. When the circadian rhythm is disturbed, or when the individual processes are abnormal during sleep, it may result in a variety of disorders.

One such example is delayed sleep-phase syndrome which is characterized by severe sleeponset insomnia. Normally, sleep is impossible until 3a.m. or later until there is great difficulty in awakening in the mornings at the normal time. The ability to cope up with circadian rhythm disturbances also differs from person to person. Identification of the individual variation would be of importance in dealing with certain sleep disorders.

\section{CHRONOPHARMACOKINETICS ${ }^{[2,3]}$}

Chronokinetics refers to dosing-time, i.e., rhythm-dependent, differences in the absorption, distribution, metabolism, and elimination of medication. Circadian rhythms in gastrointestinal $\mathrm{pH}$ can affect drug dissolution, and circadian rhythms in gastric emptying, motility, and blood flow can affect the rate, and in certain cases the amount, of drug absorption 


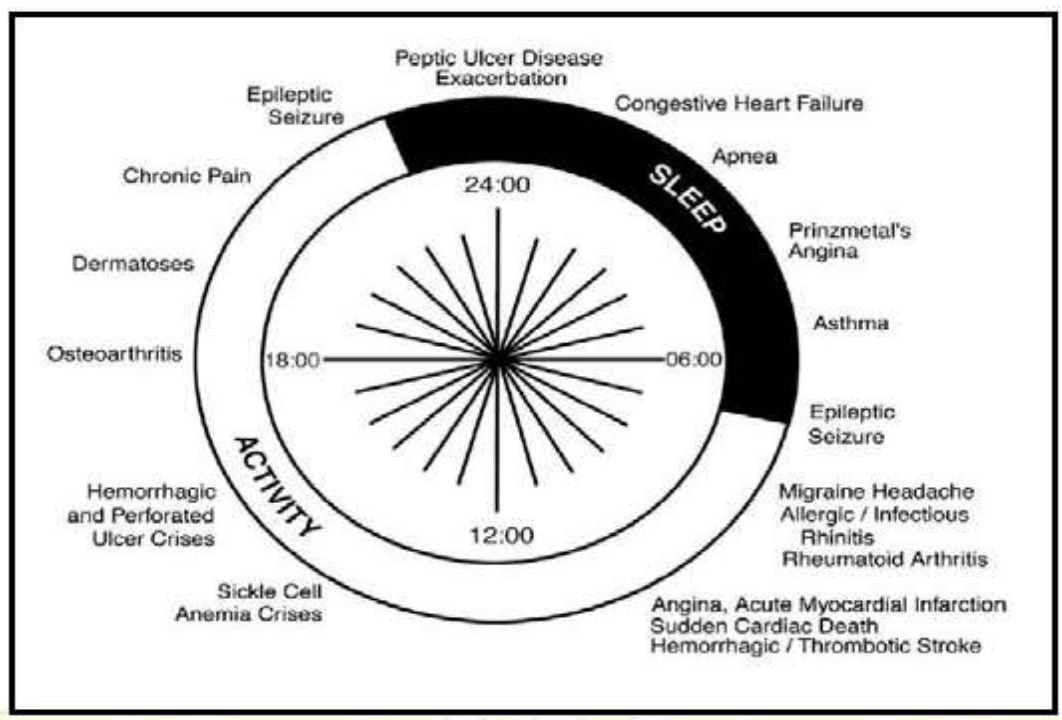

Figure 3: Display in the form of a $24 \mathrm{~h}$ clock diagram of the approximate time, in human following the diurnal activity/nocturnal sleep routine, when symptoms or events of diseases are worst or most frequently ${ }^{[2,3]}$.

Moreover, circadian rhythms in hepatic blood flow and enzyme activity can significantly affect drug biotransformation and metabolism, and rhythms in hepatic bile function and flow as well as renal blood flow, glomerular filtration, and tubular function can affect drug elimination. The pharmacokinetics, for example, the parameters of the time to peak concentration, peak height, elimination rate, volume of distribution and area under the timeconcentration curve, of a number of medications have been found to be influenced by circadian rhythms.

\section{Drug Absorption:}

In humans, for orally administered drugs, absorption was shown to be affected by CIRCADIAN rhythm as gastric acid secretion and gastric $\mathrm{pH}$, gastric motility, gastric emptying time, and gastrointestinal blood flow vary according to the time of day. These changes may have an impact on the time-dependent difference of drug absorption. For instance, circadian changes in $\mathrm{pH}$ may affect drug ionization according to its physicochemical properties. On the other hand, gastric emptying time is another important factor in the absorption of drugs. Gastric emptying rates were compared between morning (8am) and evening (8pm) in male subjects and it was found that gastric emptying $t_{1 / 2}$ for the evening meal was significantly longer for solids but not for liquids compared with those of the morning meal. The increase in evening meal gastric emptying time may also cause a delay in reaching peak plasma concentrations for several drugs. Such variations may be related to the physicochemical properties of a drug, since most lipophilic drugs seem to be absorbed faster in the morning as compared to evening. The mechanisms underlying the chronokinetics of lipophilic drugs involve a faster gastric emptying time and a higher 
gastrointestinal perfusion in the morning. However, such changes have not been shown for hydrophilic drugs. Feeding conditions also contributes to dosing time-dependent difference in drug absorption. Drug absorption by other than oral route of administration is also influenced by biological rhythms.

\section{For example,}

Skin permeability shows circadian time-dependent difference. For instance, in children, the skin penetration of an eutectic mixture of lidocaine (lignocaine) and prilocaine was reported to depend on the time of administration, with a higher rate of penetration occurring in the evening. Ocular absorption of topically applied beta-blockers is also demonstrated to be circadian time-dependent difference.

\section{Drug distribution:}

Circadian changes in biological fluids and tissues related to drug distribution are documented to vary according to time of day. Blood flow depends on several regulatory factors including sympathetic and parasympathetic systems which activities are known to be circadian time dependent with a predominant diurnal effect of the sympathetic system. Thus, diurnal increase and nocturnal decrease of blood flow may explain a possible difference in drug distribution depending on dosing time. Plasma proteins such as albumin or alpha 1 glycoprotein acid have been documented to be circadian time-dependent.

The plasma concentrations of albumin and alpha 1 glycoprotein acid show peak around noon. As a result, daily variations have been reported for drug protein binding. Temporal variations in plasma drug binding may have clinical implications only for drugs that are characterized by a high protein binding (>80\%) and a small apparent Vd.

\section{Drug Metabolism:}

Hepatic drug metabolism is generally assumed to depend on liver enzyme activity and/or hepatic blood flow: both have been shown to be circadian time-dependent Enzyme activities show circadian time-dependent difference in many tissues such as brain, kidney, and liver. Conjugation, hydrolysis and oxidation were shown to be circadian time dependent. For drugs with a high extraction ratio, hepatic metabolism depends on hepatic blood flow. Circadian variations in hepatic blood flow induce changes in liver perfusion and, thus, temporal variations in the clearance of such drugs. Clearance in healthy volunteers shows the highest values in the early morning.

\section{Drug excretion:}

Renal physiological functions such as glomerular filtration, renal blood flow, urinary $\mathrm{pH}$ and tubular resorption show circadian time-dependent difference with higher values during daytime. These rhythmic variations in renal functions may contribute to circadian dependent 
change in drug urinary excretion. The rhythmicity in urinary $\mathrm{pH}$ modifies drug ionization and may explain that acidic drugs are excreted faster after an evening administration as demonstrated for sodium salicylate and sulfasalazine. Such variations are obviously more pronounced for hydrophilic drugs. The circadian timing system plays a key role in the changes of toxicity of drugs by influencing their metabolisms in the liver and intestine in addition to their excretion via bile flow and urine. Rats with chronic biliary drainage under a rigid lighting schedule (light on at 6 am and off at $6 \mathrm{pm}$ ) exhibited a remarkable circadian rhythm of bile flow, biliary concentrations and excretory rates of bile salts, cholesterol and phospholipids. On the other hand, the excretion rates of these polyamines were found to highest in the morning in healthy volunteer subjects.

Table 2: Possible physiological factors influencing circadian stage-dependent pharmacokinetics of drug ${ }^{[2,3]}$.

\begin{tabular}{l}
\hline Absorption \\
\hline Oral: Gastric pH, Gastric motility, Gastric emptying time, Gastrointestinal blood flow, transporter. \\
Parenteral: Transdermal permeability, Ocular permeability, Pulmonary permeability \\
\hline Distribution \\
\hline Blood flow, albumin, $\alpha 1$-acid glycoprotein, red blood cells, transporter. \\
\hline Metabolism \\
\hline Liver enzyme activity, hepatic blood flow, gastrointestinal enzyme. \\
\hline Elimination \\
\hline $\begin{array}{l}\text { Renal, Biliary, Intestinal, Glomerular filtration, Renal blood flow, Urinary pH, Electrolytes, } \\
\text { Tubular resorption, }\end{array}$ \\
\hline
\end{tabular}

\section{Chronodynamics ${ }^{[2]}$}

Chronodynamics refers to dosing-time, i.e., rhythm-dependent, differences in the effects of medications. Such administration time differences are due to rhythms in the free-to-bound drug fraction, number and conformation of drug-specific receptors, second messenger and ion channel dynamics, and rate-limiting step(s) in metabolic pathways. Both the desired/ beneficial and undesired/adverse effects of medications can vary significantly according to their administration time. ${ }^{28}$ Non-steroid class of anti-inflammatory drugs (NSAIDs), are prescribed to manage the pain, stiffness, and inflammation of rheumatoid and osteoarthritis. A number of clinical trials with NSAIDs have shown that they exert better therapeutic effect on the aggravating morning symptoms of rheumatoid arthritis, and are better tolerated, when ingested around or at bedtime than in the morning upon arising. On the hand, NSAIDs are 
more effective in reducing the afternoon and evening peak intensity of osteoarthritis symptoms when ingested in the morning or around lunch time, although the likelihood of gastric intolerance and other adverse effects is greater when they are routinely ingested in the morning as opposed to evening.

\section{Chronotoxicology ${ }^{[2]}$}

Chronotoxicology, is an aspect of chronodynamics; it refers specifically to dosing-time, i.e., rhythm-dependent, differences in the manifestation and severity of adverse effects and thus intolerance of patients to medications. Classes of medications that have high risk of adverse effects and relatively narrow therapeutic range, in particular, are likely to show significant dosing-time differences in safety. These medications are best tolerated - cause least adrenocortical suppression when ingested as a single daily dose in the morning at the commencement of the daily activity span, when a part or all of a moderate daily dose of the glucocorticoid is ingested, injected, infused, or inhaled late in the day, especially in the evening between dinner and bedtime, the risk of adrenocortical suppression is heightened, even after only a few days of treatment. These chronotoxicological findings have significantly impacted the manner in which synthetic corticosteroids are used in clinical practice. These and other findings from both human and laboratory animal studies imply that drug-delivery systems must be designed to minimize the potential risk of adverse effects of pharmacotherapies by taking into consideration their specific circadian chronotoxicologies.

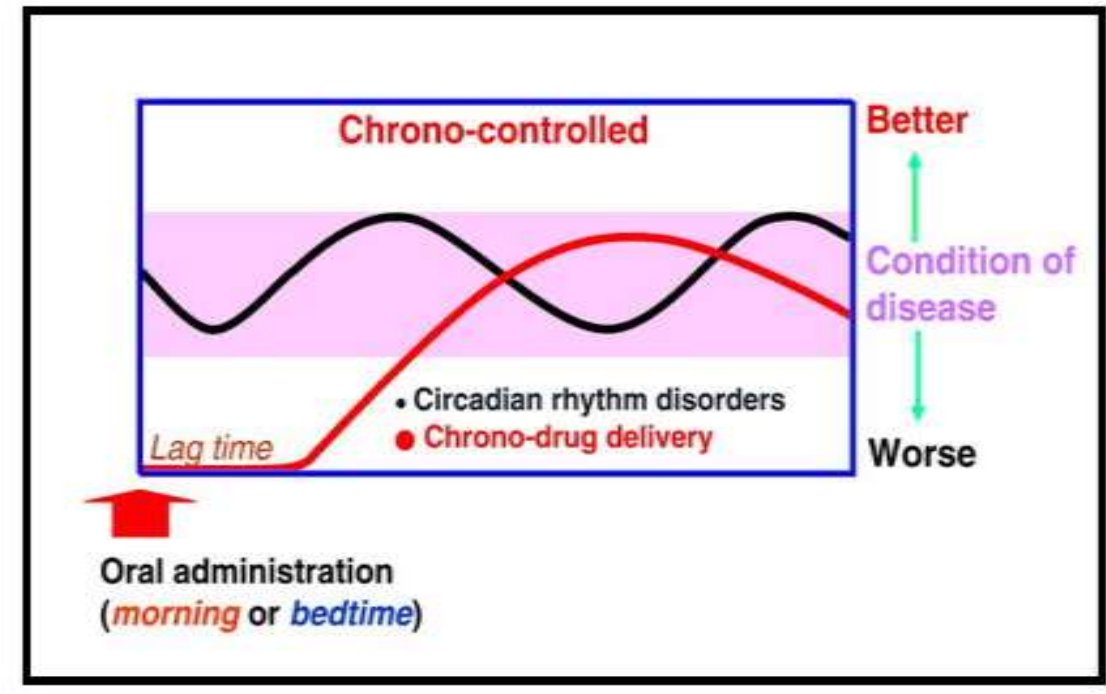

Figure 4: Circadian rhythm disorders and chrono drug delivery ${ }^{[2]}$.

Table 3: Predictable circadian variation can be useful in diagnosis of various diseases ${ }^{[2]}$.

\begin{tabular}{lll}
\hline Sl. No & Diseases/Syndrome & Circadian rhythmicity \\
\hline 1 & Allergic rhinitis & Symptoms worse in early morning \\
2 & Bronchial asthma & Exacerbations more common during the Sleep period \\
3 & Arthritis rheumatoid & Symptoms are most intense rheumatoid upon awakening \\
4 & Osteoarthritis & Symptoms worse in the middle/latter portion of the day \\
\hline
\end{tabular}




\begin{tabular}{lll}
\hline 5 & Anti cancer agents & Doxorubicin, Cisplatin, methotrexate \\
6 & NSAIDs & Ibuprofen, Indomethacin, Tenoxicam, Acetylsalicylic acid \\
7 & Angina pectoris & Chest pain and ECG changes more common during the early morning \\
8 & Myocardial infarction & Incidence greatest in the infarction early morning \\
9 & Peptic ulcers & $\begin{array}{l}\text { Symptoms worse after gastric emptying and in the early morning (sleep } \\
\text { period) }\end{array}$ \\
10 & Stroke & Incidence greatest in early morning \\
11 & Epilepsy & Incidence greatest in early morning \\
\hline
\end{tabular}

\section{CHRONOPHARMACOKINETICS OF DRUGS ${ }^{[3]}$}

\section{Antiulcer Drugs:}

H2-blockers (ranitidine, cimetidine, famotidine, roxatidine, nizatidine) should be taken once a day in the late afternoon or early night when acid secretion is increasing, independently of whether the compounds have a short or a long half-life. In contrast to H2-blockers proton pump inhibitors (PPI) should be dosed in the morning since the increase by lansoprazole and omeprazole in intragastric $\mathrm{pH}$ is more pronounced after morning than evening administration.

\section{Antihypertensive drugs:}

Cardiovascular drugs such as nifedipine, oral nitrates and propranolol, plasma peak concentration is twice as high and time to reach peak concentration is shorter after morning dosing compared with evening dosing. Such a variation was not detected when sustained release dosage forms of nifedipine and isosorbide mononitrate were used. The underlying mechanisms of their chronopharmacokinetic pattern involve a faster gastric emptying time and a greater gastrointestinal perfusion in the morning. Shiga et al documented that atenolol, in contrast to propranolol, is not absorbed more rapidly after morning administration compared with post-evening administration. This confirms that the absorption rate of a lipophilic, but not hydrophilic, drugs is faster after morning dosing

\section{Anti-inflammatory drugs:}

Studies on NSAIDs, e.g., indomethacin and ketoprofen, have also shown that these drugs have a greater rate and/or extent of bioavailability when they are given in the morning than when they are given in the evening due to better morning absorption. Earlier and higher peak concentrations were obtained when ketoprofen and indomethacin was given at 07:00 and 07:00 or 11:00 respectively than at other times of the day or night. Greater blood flow of the gastrointestinal tract in the morning than in the evening may explain this phenomenon. Circadian changes in renal function, plasma protein binding or hepatic blood flow could also explain temporal variation in drug plasma levels. Many variables are known to influence pharmacokinetics.

\section{Antiasthmatic Drugs:}


Antiasthmatic drugs such as theophylline and beta sympathomimetics may/ should be dosed higher in the evening than during daytime when asthma is predominantly nocturnal. The theophylline concentration peak height $(\mathrm{Cmax})$ is greater and time to peak (Tmax) shorter with dosing at 08:00 than at 20:0015. Both the first and second-generation H1- receptor antagonists of cyproheptadine, terfenadine, clemastine, and mequitazine exerted a statistically significantly longer duration of action when they were ingested in the morning (7 a.m.) than evening (7 p.m.).

\section{Anti-Migraine drugs:}

Sumatriptan is a drug of choice in migraine treatment. The meanpeak serum concentration following the $0700 \mathrm{~h}$ administration was significantly higher than after the $1900 \mathrm{~h}$ administration. AUC and AUMC were significantly higher following the 0700 and $0100 \mathrm{~h}$ administrations than after the $1900 \mathrm{~h}$ administration. Following administration at $0700 \mathrm{~h}$, the mean oral clearance and the apparent volume of distribution were significantly lower than after the $1900 \mathrm{~h}$ administration.

\section{Antibiotics:}

Administration-time-dependent differences in the pharmacokinetics and toxicity of antimicrobial agents have been documented in Table 4. This is particularly true for the aminoglycosides, as their nephrotoxicity is greatest when administered during the resting period. Food intake and low urinary $\mathrm{pH}$ has been found to be protective of the toxicity of aminoglycosides at this time of the day16. Knowledge of the administration-time-dependence of aminoglycosides and the underlying mechanisms can be used to develop once-a-day formulations that are significantly less toxic, in particular to the kidney, in patients who require around-the-clock antimicrobial therapy.

Table 4: Circadian variations in the pharmacokinetics of antibiotics in human beings ${ }^{[3]}$.

\begin{tabular}{|c|c|}
\hline Results & Antibiotics \\
\hline Higher mean serum levels at 5 a.m. & Netilmicin \\
\hline \multicolumn{2}{|l|}{ Higher trough serum levels at 9 a.m. } \\
\hline Higher serum levels at 9 p.m. & Amikacin \\
\hline \multicolumn{2}{|c|}{ Longer serum half-life between $8 \mathrm{pm}$. and midnightGentamicin } \\
\hline Lower renal clearance between $8 \mathrm{p}$. & and \\
\hline Midnight & isepamicin \\
\hline \multicolumn{2}{|c|}{ Higher AUC (serum) between 8 p.m. and midnight } \\
\hline Lower renal clearance at 8 p.m. & Amikacin \\
\hline \multicolumn{2}{|c|}{ Higher renal cortex accumulation at 1:30 a.m. } \\
\hline Lower renal clearance at 1:30 a.m. & \\
\hline Higher AUC (serum) at midnight & Cefodizime \\
\hline Longer serum half-life at midnight & Sulfamethoxazole \\
\hline Lower urine excretion at 10 p.m. & Ciprofloxine \\
\hline $\begin{array}{l}\text { No significant circadian difference in } \\
\text { serum half-life and renal clearance }\end{array}$ & Netilmicin \\
\hline
\end{tabular}




No significant circadian difference in renal Vancomycin
Clearance

\section{Anticancer Drugs:}

In human bone marrow, skin, and oral and rectal mucosae, DNA synthesis, a stage of the celldivision cycle associated with increased susceptibility to Sphase-specific agents, decreases by $50 \%$ or more between 00:00 and 04:00 compared with daytime. The activity of dehydropyrimidine dehydrogenase in human mononuclear cells increases by $40 \%$ around midnight. This enzyme brings about the intracellular catabolism of 5-FU and contributes to improved tolerability of this drug between 00:00 and 04:00. In contrast, cisplatin are better tolerated between 16:00 and 20:00 than $12 \mathrm{~h}$ apart. The chronopharmacokinetic finding of cisplatin seems to contribute to the decreased renal toxicity during evening administration. These findings show that the circadian stage at which anticancer drugs are given to patients should be carefully considered. One approach to increasing the efficiency of pharmacotherapy is administering drugs at times during which they are best tolerated given in Table 5 .

Table 4: Circadian variations in the pharmacokinetics of antibiotics in human beings ${ }^{[3]}$.

\begin{tabular}{|c|c|c|}
\hline \multicolumn{2}{|l|}{ Results } & Antibiotics \\
\hline \multicolumn{2}{|l|}{ Higher mean serum levels at 5 a.m. } & Netilmicin \\
\hline \multicolumn{3}{|l|}{ Higher trough serum levels at 9 a.m. } \\
\hline \multicolumn{2}{|l|}{ Higher serum levels at 9 p.m. } & Amikacin \\
\hline \multicolumn{2}{|c|}{ Longer serum half-life between $8 \mathrm{pm}$. and midnight } & Gentamicin \\
\hline \multicolumn{2}{|c|}{ Lower renal clearance between 8 p.m. and Midnight } & And isepamicin \\
\hline \multicolumn{3}{|c|}{ Higher AUC (serum) between 8 p.m. and midnight } \\
\hline \multicolumn{2}{|c|}{$\begin{array}{l}\text { Lower renal clearance at } 8 \text { p.m. Higher renal cortex } \\
\text { accumulation at 1:30 a.m. Lower renal clearance at 1:30 a.m. }\end{array}$} & m. Amikacin \\
\hline \multicolumn{2}{|l|}{ Higher AUC (serum) at midnight } & Cefodizime \\
\hline \multicolumn{2}{|l|}{ Longer serum half-life at midnight } & Sulfamethoxazole \\
\hline \multicolumn{2}{|l|}{ Lower urine excretion at 10 p.m. } & Ciprofloxine \\
\hline \multicolumn{3}{|c|}{$\begin{array}{l}\text { No significant circadian difference in serum half-life and renal Netilmicin } \\
\text { clearance }\end{array}$} \\
\hline \multicolumn{2}{|c|}{ No significant circadian difference in Renal Clearance } & Vancomycin \\
\hline \multicolumn{3}{|c|}{ Table 5: Tolerance to Circadian-based Cancer Chemotherapy in Humans } \\
\hline \multicolumn{2}{|l|}{ Drug } & $\begin{array}{l}\text { Time of optimal host } \\
\text { tolerance }\end{array}$ \\
\hline \multirow[t]{2}{*}{ Doxorubicin } & Ovarian Bladder & $06.00 \mathrm{hr}(>18.00 \mathrm{hr})$ \\
\hline & & $06.00 \mathrm{hr}(>18.00 \mathrm{hr})$ \\
\hline 4-Tetrahydropyranyl adriamycin & Ovarian & $06.00 \mathrm{hr}(>18.00 \mathrm{hr})$ \\
\hline Cisplatin & Ovarian Bladder & $\begin{array}{l}16.00 \mathrm{hr}-20.00 \mathrm{hr} \\
(>4.00-08.00 \mathrm{hr})\end{array}$ \\
\hline Etoposide & Solid Tumors & $07.00 \mathrm{hr}(>19.00 \mathrm{hr})$ \\
\hline $5-\mathrm{FU}$ & & $02.00-10.00 \mathrm{hr}$ \\
\hline Methotrexate & & $08.00 \mathrm{hr}$ \\
\hline Anthracyclins & & early morning \\
\hline
\end{tabular}




\section{Opioid analgesics:}

Stronger analgesic effects were observed when tramadol and dihydrocodeine were applied in the evening to relieve painful stimuli. Peak morphine use occurred at 9 a.m. and least use at 3 a.m. In postoperative patients undergoing elective cholecystectomies, the need for fentanyl was lower when the surgery was done between 8 and 10 a.m. than between 11 a.m. and 3 p.m. Finally, a recent study of meperidine reveals a circadian variation of meperidine-induced analgesia in sickle cell anemia patients, with maximal analgesic effect occurring with the morning dose. A prospective study reported the existence of circadian variation in the distribution of lethal opiate overdoses in drug abusers, with a high death risk in the evening hours (03:00-09:00 h).

\section{Local anesthetics:}

The duration of local anesthesia was longest when amide-type local anesthetic agents (lidocaine, ropivacaine, mepivacaine and betoxycaine) were applied around 3 p.m. Area under the lidocaine plasma concentration curves (AUC) was largest in the afternoon. A $60 \%$ change was found in the 24-hour plasma clearance of bupivacaine, the clearance being greatest at 6 a.m. An administration-time-dependent variation in the transcutaneous passage of a medication was also studied. The plasma levels of lidocaine were significantly higher in the evening than at any other time of day.

\section{Immunosupressants:}

A slightly increased AUC and AUMC resulting from decreased apparent clearance during the resting (PM) versus activity (AM) period were observed for cyclosporine. A significant delay in mean residence time was observed after the PM dose, and the PM area under the moment curve was larger than the AM value. These trends and differences suggest that more sophisticated time-dependent cyclosporine dosing methods are needed to balance AM and PM drug exposure and thereby improve immunosuppression.

\section{CHRONOTHERAPEUTIC DRUG DELIVERY SYSTEMS ${ }^{[4]}$}

The effectiveness and toxicity of many drugs vary depending on the relationship between the dosing schedule and the $24 \mathrm{~h}$ rhythms of biochemical, physiological and behavioral processes. In addition, several drugs can cause alterations to the $24 \mathrm{~h}$ rhythms leading to illness and altered homeostatic regulation. The alteration of biological rhythm is a new concept of adverse effects. The disruption of circadian rhythm can be minimized by optimizing the dosing schedule. The major aim of chronopharmaceutics is to deliver the drug in higher concentrations during the time of greatest need and in lesser concentrations when the need is less to minimize side effects. Various technologies to develop time-controlled peroral drug delivery systems have been extensively studied in recent decades. Some of these 
systems are discussed in the following subsections.

\section{Enteric-coated systems:}

Enteric coatings have traditionally been used to prevent the release of a drug in the stomach (see Figure 5). Enteric coatings are $\mathrm{pH}$-sensitive and drug is released when $\mathrm{pH}$ is raised above 5 in the intestinal fluid. These formulations can be utilised in time-controlled drug administration when a lag time is needed. Because of the unpredictability of gastric residence, such systems cannot be the first choice when a time-controlled release is required. In the treatment of nocturnal asthma, a salbutamol formulation containing a barrier coating which is dissolved in intestinal $\mathrm{pH}$ level above about 6 , has been successfully used. The system contains a core which is film coated with two polymers, first with HPMC and then with a gastro-resistant polymer (Eudragit ${ }^{\circledR}$ L30D). In this system the duration of the lag phase in absorption can be controlled by the thickness of the HPMC layer.

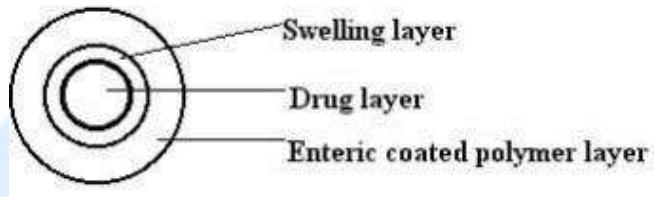

Figure 5: Schematic representtion of enteric coated system ${ }^{[4]}$.

\section{Layered systems:}

These are one or two impermeable or semi-permeable polymeric coatings (films or compressed) applied on both sides of the core. To allow biphasic drug release, a three-layer tablet system was developed. The two layers both contain a drug dose. The outer drug layer contains the immediately available dose of drug. An intermediate layer, made of swellable polymers, separates the drug layers. A film of an impermeable polymer coats the layer containing the other dose of drug. The first layer may also incorporate a drug-free hydrophilic polymer barrier providing delayed $(5 \mathrm{~h})$ drug absorption. Conte et al has also studied a multilayer tablet system (Geomatrix ${ }^{\circledR}$ ). It consists of a hydrophilic matrix core containing the drug dose. This kind of three- layer device has been used in the treatment of Parkinsonism patients using L--dopa/benserazide. Night-time problems and early-morning symptoms of Parkinsonism can be avoided by using a dual-release Geomatrix@ formulation, which allows daily doses of drug to be reduced and leads to extent of bioavailability $40 \%$ greater than when a traditional controlled release formulation is employed.

\section{Time-controlled explosion systems (TES):}

These have been developed for both single and multiple unit dosage forms. In both cases, the core contains the drug, an inert osmotic agent and suitable disintegrants. Individual units can be coated with a protective layer and then with a semi-permeable layer, which is the rate 
controlling membrane for the influx of water into the osmotic core. As water reaches the core, osmotic pressure is built up. The core ultimately explodes, with immediate release of the drug. The explosion of the formulation can also be achieved through the use of swelling agents. Lag time is controllable by varying the thickness of the outer polymer coating.

\section{Sigmoidal release systems (SRS):}

For the pellet-type multiple unit preparations, SRS containing an osmotically active organic acid have been coated with insoluble polymer to achieve different lag-times. By aplying different coating thicknesses, lag times in vivo of up to 5 hours can be achieved. Release rates from SRS, beyond the lag time, has been found to be independent of coating thickness.

\section{Press-coated systems:}

Delayed-release and intermittent-release formulations can be achieved by press-- coating. Press-coating, also known as compression coating, is relatively simple and cheap, and may involve direct compression of both the core and the coat, obviating the need for a separate coating process and the use of coating solutions. Materials such as hydrophilic cellulose derivatives can be used and compression is easy on a laboratory scale. On the other hand, for large-scale manufacture, special equipment is needed. The major drawbacks of the technique are that relatively large amounts of coating materials are needed and it is difficult to position the cores correctly for the coating process.

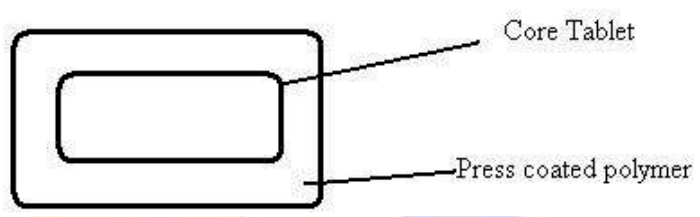

Figure 6: Schematic representation of a press-coated system ${ }^{[4]}$.

In recent years, various controlled release, especially time-controlled release, drug delivery systems based on compression coating technology have been studied. Most of such formulations release drug after a lag phase, followed by a rapid dissolution of the core. Conte et al have developed a press-coated device in which the inner core contains the drug and the outer coat is made of different types of polymers. The outer barrier, which controls drug release, can be either swellable or erodible. Lag times can be varied by changing the barrier formulation or the coating thickness. Matsuo et al have developed a diltiazem hydrochloride formulation intended for use in the treatment of time-related symptoms of ischaemic heart disease and hypertension. The tablet consists of a core, which contains the drug, and a coat formed by compressing hydroxyethylcellulose. Diltiazem is rapidly released after a delay of several hours. Marvola et al have developed a press-coated tablet formulation in which most of the total amount of drug is in the tablet core. Hydrophilic polymers such as hydroxypropyl 
methylcellulose and sodium alginate have been used in the coat to control drug release as illustrated in Figure 7. The extent of bioavailability of furosemide, ibuprofen and salbutamol sulphate from the system developed has been found to be satisfactory.

\section{Capsular Systems}

It consists of drug formulation inside a plug which is erodible after a predetermined lag phase along with an outer coating of a water insoluble capsule. A swellable hydrogel plug closes the open end of the capsule body. As the capsule comes in contact with fluids the plug swells after the predetermined lag phase and comes out of the capsule leading to the pulsatile release of the drug. The plug is mainly formed by permeable and soluble polymers such as HPMC, agar, pectin and polymetaacrylates. The best example of developed capsular system would be pulsincap system (Figure $8 \& 9$ ). ${ }^{1}$

\section{Rupturable Coating Systems}

In such kind of systems coating ruptures or disintegrates to release a particular drug. Coating ruptures due to swelling/ osmotic pressure/disintegration/effervescent recipient. The effervescent mixture is generally composed of citric acid and borax which is inserted into the core further coated with ethyl cellulose. Pressure generated due to the formation of the carbon dioxide gas leads to the rupturing of the coating. Increased coating thickness and increased
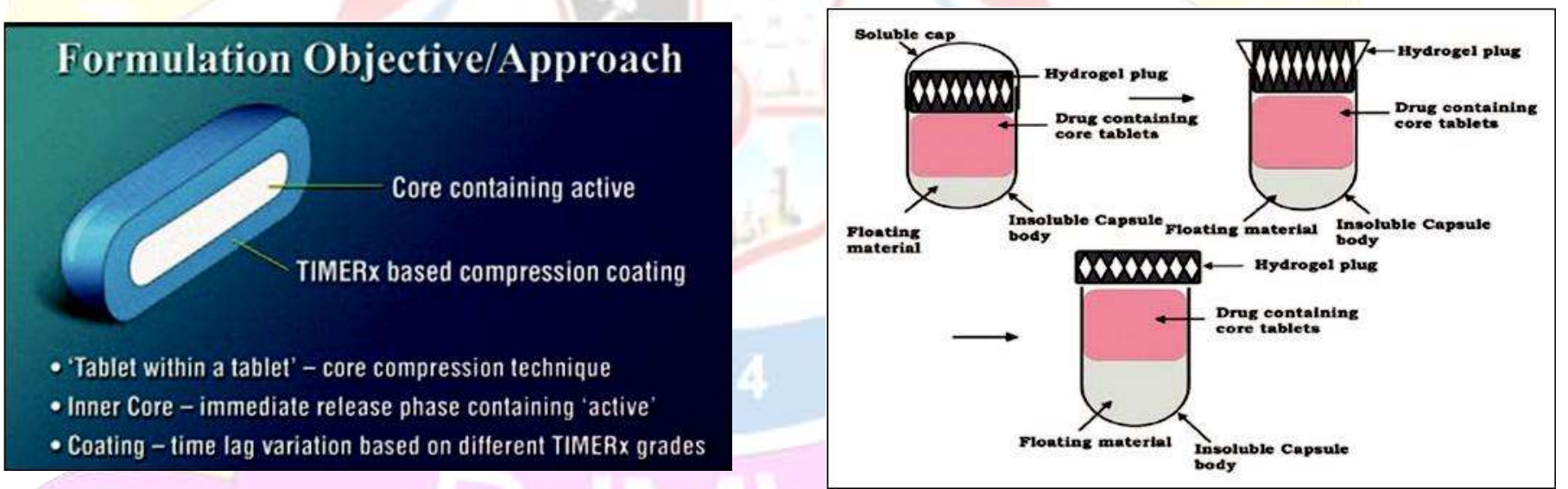

Figure 7: Formulation approach for single unit system ${ }^{[1]}$ Figure 8: Capsular system ${ }^{[1]}$. hardness of the core tablet leads to the increase in the lag time. Certain agents such as sodium starch glycollate and low substituted hydroxyl propyl cellulose are used as the swelling agents and they swell upon contact with the GI fluids leading to the complete film rupture and resultant drug release. 


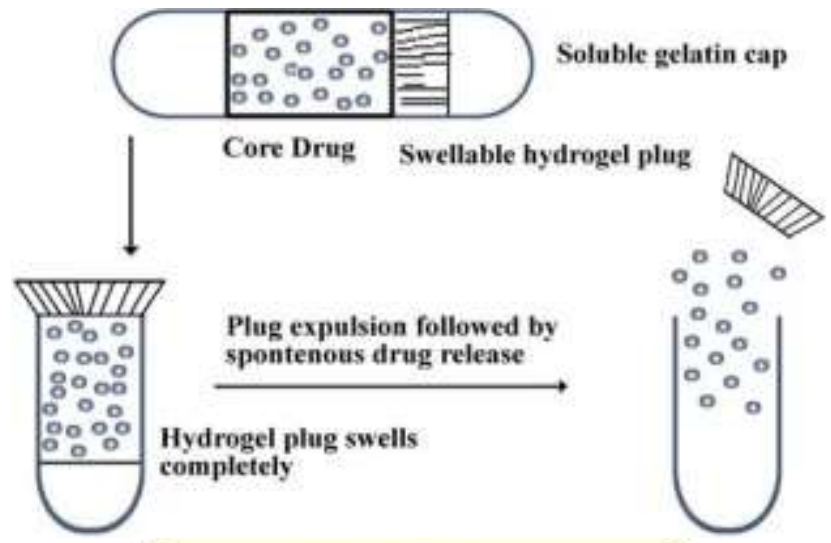

Figure 9: Schematic diagram of release from pulse in cap

\section{Osmosis Based Capsular System (Port System)}

It consists of a semi permeable membrane coating a gelatine capsule. Osmotically active agents present in the capsule inside an insoluble plug within the capsule. As this capsule comes in contact with the oral and GI fluids the water diffuses across the semi permeable membrane resulting in increased pressure that results in resultant release of the drug a particular predetermined lag time.

Eg: Ritalin (methyl phenidate): Attention Deficit Hyperactive Disorder

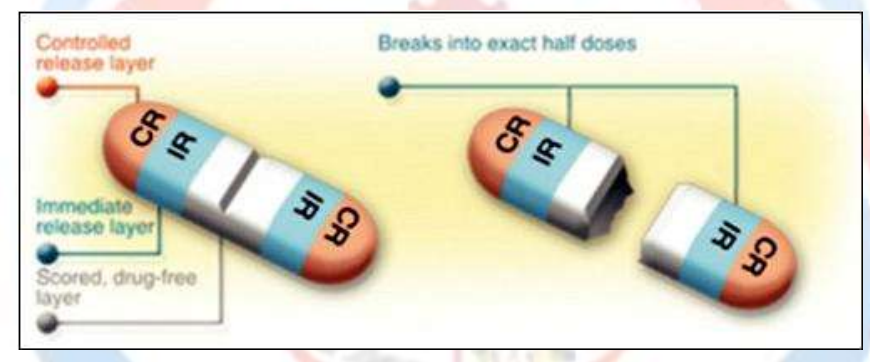

Figure 10: DMDS technology ${ }^{[1]}$.

\section{Soluble Barrier Coating System}

Here a barrier membrane coats the reservoir of the drug and barrier dissolves after a specific lag time leading to the chronotropic release of the drug. Mainly in the chronotropic system core consists of a coating by HPMC a hydrophilic swellable polymer or cellulose acetate phthalate which results in desired lag phase of the drug release.

\section{Multiparticulate Sytems}

They are generally in the form of beads and pellets and they mainly act as reservoirs. All the granules are packed in a capsule after coated the drug over sugar beads. The main advantage of such kind of systems is that it prevents the dose dumping. There are few kinds of multiparticulate system mainly categorized on the basis of pulsatile release by osmotic rupture or rupture of membrane due to other reasons.

Examples ${ }^{[1,3]}$ of Chrono-DDS on the market, are given in Table 5. Most data have been compiled from FDA electronic orange book, specific product package inserts and United 
States patents and specific pharmaceutical company websites.

\section{CONTIN® technology:}

In this technology, molecular coordination complexes are formed between a cellulose polymer and a non-polar solid aliphatic alcohol optionally substituted with an aliphatic group by solvating the polymer with a volatile polar solvent and reacting the solvated cellulose polymer directly with the aliphatic alcohol, preferably as a melt. This constitutes the complex having utility as a matrix in controlled release formulations since it has a uniform porosity (semipermeable matrixes) which may be varied.

E.g.: Evening administration of Uniphyl ${ }^{\circledR}$ (anhydrous theophylline) tablets represented a rational dosing schedule for patients with asthma who often exhibit increased bronchoconstriction in the morning.

\section{Physico-chemical modification of the API:}

In this strategy, a proprietary method is used to modify the physicochemical properties (e.g. solubility, partition coefficient, membrane permeability, etc.) of the API to achieve the chronopharmaceutical objective. Typical examples of the use of this strategy in chronotherapy are those of antihyperlipidemic statins (HMG-CoA reductase inhibitors) and antiulcerative agents (histamine $\mathrm{H} 2$ receptor-antagonists).

\section{OROS® technology:}

OROS $®$ technology uses an osmotic mechanism to provide pre-programmed, controlled drug delivery to the gastrointestinal tract.

Ex: OROS® Delayed Push- Pull System, also known as controlled onset extended release (COER) was used to design Covera- HSR, a novel anti hypertensive product. It actually enabled delayed, overnight release of verapamil to help prevent the potentially dangerous surge in BP that can occur in the early morning.

\section{CODAS ${ }^{\circledR}$ technology:}

The Chronotherapeutic Oral Drug Absorption System (CODAS®) is a multiparticular system which is designed for bedtime drug dosing, incorporating a 4- $5 \mathrm{~h}$ delay in drug delivery. This delay is introduced by the level of non-enteric release-controlling polymer applied to drug loaded beads.

E.g.: The CODAS®- verapamil extended release capsules (Verelan® PM) as ChrDDS actually provided enhanced BP reduction during the morning period when compared with other time intervals of the $24-\mathrm{h}$ dosing period.

\section{CEFORM® technology:}

The CEFORM® technology allows the production of uniformly sized and shaped microspheres of pharmaceutical compounds. This ChrDDS approach is based on "melt- 
spinning", which means subjecting solid feedstock i.e. biodegradable polymer/bioactive agents combinations to the combination of temperature, thermal gradients, mechanical forces, flow, and flow rates during processing. The microspheres may be coated for controlled release either with an enteric coating or combined into a fast/ slow release combination.

E.g.: CardizemR LA, 1-day diltiazem formulation as ChrDDS.

\section{DIFFUCAPS® technology:}

In the DIFFUCAPS ${ }^{\circledR}$ technology, a unidosage form, such as a capsule for delivering drugs into the body in a circadian release fashion, is comprising of one or more populations of drug-containing particles (beads, pellets, granules, etc..). Each bead population exhibits a pre designed rapid or sustained release profile with or without a predetermined lag time of $3-5 \mathrm{~h}$. E.g.: Propranolol-containing ChrDDS (InnopranR XL) for the management of hypertension.

\section{Chronomodulating infusion pumps:}

Externally and internally controlled systems across a range of technologies including preprogrammed systems, as well as systems that are sensitive to modulated enzymatic or hydrolytic degradation, $\mathrm{pH}$, magnetic fields, ultrasound, electric fields, temperature, light and mechanical stimulation have been developed.

E.g.: The Melodie ${ }^{\circledR}$, programmable Synchromed ${ }^{\circledR}$, Panomat ${ }^{\circledR}$ V5 infusion, and the Rhythmic ${ }^{\circledR}$ pumps.

\section{TIMERx$®$ technology:}

The TIMERx ${ }^{\circledR}$ technology (hydrophilic system) combines primarily xanthan and locust bean gums mixed with dextrose. The physical interaction between these components works to form a strong, binding gel in the presence of water. Drug release is controlled by the rate of water penetration from the gastrointestinal tract into the TIMERx® gum matrix, which expands to form a gel and subsequently releases the active drug substance.

E.g.: An oral, CR opioid analgesic oxymorphone.

\section{Three-dimensional printing:}

Three dimensional printing® (3DP) is a novel technique used in the fabrication of complex oral dosage delivery pharmaceuticals based on solid freeform fabrication methods. It is possible to engineer devices with complicated internal geometries, varying densities, diffusivities, and chemicals. Different types of complex oral drug delivery devices have been fabricated using the 3DP process: immediate-extended release tablets, pulse release, breakaway tablets, and dual pulsatory tablets.

\section{PRODAS technology:}

PRODAS stands for Programmable Oral Drug Absorption system. It mainly focuses on uniting the tablet technology within a capsule as a multi particulate system in order to control 
the drug release.

\section{DMDS (Dividable Multiple Action Delivery Systems) Technology (Figure 10):}

It mainly focuses improving drug efficacy by allowing the drug tablet to be broken into two halves each being released in order to achieve the same rate profile of that of the whole tablet at different time thereby reducing the side effects and the ease of the adjustment of the dosage.

\section{ACCU-BREAK Technology:}

They focus on divisible tablets which result in exact smaller dose post division. They contain a controlled release medication separated by drug free break layer.

\section{SODAS (Spheroidal Oral Drug Absorption System) Technology:}

It is a multi particulate system that enables the drug to be released in pulsatile bursts throughout the day. It mainly has spheroidal beads of $2 \mathrm{~mm}$ diameter coated with polymers for controlled release.

\section{Other CR erodible polymers:}

Erodible polymers have been designed in different forms (e.g. tablets, capsules, micro particles) for ChrDDS applications. An excellent review of pulsatile drug-delivery system involving erodible polymers has been made by Bussemer et al. By careful selection and combination of polymeric drug carrier of different erosion/degradation kinetic, or by manipulating the interaction energy between the drug and the polymer, it may be possible to control the release of a drug at a rate that matches the requirement of the biological rhythm of a given disease state.

\section{Controlled-release microchip:}

An alternative method to achieve pulsatile or chronopharmaceutical drug release involves using micro fabrication technology. Santini et al.33 reported a solid-state silicon microchip that can provide controlled release of single or multiple chemical substances on demand.

Table 6: Examples of chrono-drug delivery system on the market in USA and Japan ${ }^{[3]}$

\begin{tabular}{|c|c|c|c|}
\hline $\begin{array}{l}\text { Active } \\
\text { pharmaceutical } \\
\text { ingredient (API) }\end{array}$ & $\begin{array}{l}\text { Proprietary name } \\
\text { dosages form }\end{array}$ & $\begin{array}{l}\text { Proprietary } \\
\text { chronopharmaceutical } \\
\text { technology }\end{array}$ & Disease \\
\hline USA & 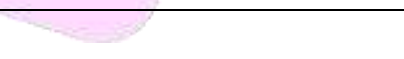 & & \\
\hline Theophylline & $\begin{array}{l}\text { Uniphyl@ extended } \\
\text { release tablets }\end{array}$ & CONTIN® & Asthma \\
\hline Famotidine & Pepcid® Tablets & $\begin{array}{l}\text { Physico-chemical } \\
\text { modification of API }\end{array}$ & Ulcer \\
\hline Simvastatin & Zocor ${ }^{\circledR}$ tablets & $\begin{array}{l}\text { Physico-chemical } \\
\text { modification of API }\end{array}$ & Hyperlipidemia \\
\hline Verapamil $\mathrm{HCl}$ & $\begin{array}{l}\text { Covera-HS® extended } \\
\text { release tablets }\end{array}$ & OROS® & Hypertension \\
\hline Verapamil HCl & Verelan®PM extended & CODAS $®$ & Hypertension \\
\hline
\end{tabular}




\begin{tabular}{|c|c|c|c|}
\hline Diltiazem $\mathrm{HCl}$ & $\begin{array}{l}\text { release capsules } \\
\text { Cardizem }{ }^{\circledR} \text { LA } \\
\text { extended release tablets }\end{array}$ & CEFORM® & Hypertension \\
\hline Propranolol $\mathrm{HCl}$ & InnoPran ${ }^{\circledR} \mathrm{XL}$ & DIFFUCAPS® & Hypertension \\
\hline \multicolumn{4}{|l|}{ Japan } \\
\hline Famotidine & Gaster ${ }^{\circledR}$ tablets & $\begin{array}{l}\text { Physico-chemical } \\
\text { modification of API }\end{array}$ & Ulcer \\
\hline Simvastatin & Lipovas $\AA$ tablets & $\begin{array}{l}\text { Physico-chemical } \\
\text { modification of API }\end{array}$ & $\begin{array}{l}\text { Hyperlipidemi } \\
\text { a }\end{array}$ \\
\hline Theophylline & $\begin{array}{l}\text { Uniphyl } ® \text { extended } \\
\text { release tablets }\end{array}$ & $\begin{array}{l}\text { CONTIN® Transdermal } \\
\text { chrono delivery }\end{array}$ & Asthma \\
\hline Tulobuterol & Hokunalin $®$ tape & system & Asthma \\
\hline
\end{tabular}

\section{CONCLUSION:}

Chronotheraputics has importance in drug therapy depending according to biological rhythms and this has led to a new approach to the development of drug delivery systems. If symptoms of a disease display circadian variation, drug release should also vary over time. In the recent years different technologies have been applied to develop time-controlled, pulsed, triggered and programmed drug delivery devices to get optimal clinical outcome with constant plasma concentrations. Since it is seems that timing of drug administration in disease therapy has significant impact upon treatment success, Chronotheraputics remains an important area for continuing research. It can be concluded that oral chronotropic drugs help in various drug delivery problems such as extensive first pass metabolism, chronotropic behaviour of the diseases and nocturnal dosing thereby increasing the patient compliance and is the future of the drug delivery systems.

\section{REFERENCES:}

1. Bhatia s, kumar b, Mittal s. Oral Chronotherapeutics: Future Of Drug Delivery Systems. Int J Sci Stud. 2014;2(4):55-58.

2. Chandan kumar brahma, G.Vidya Sagar gali, Chronotherapeutics Drug Delivery Systems Challenges To Pharmaceutical Field. J Global Trends in Pharm Sci 2012 volume 3, issue 3, pp -778-791.

3. N.1. Prasanthi, G. Swathi, S.S.Manikiran, International Journal of Pharmaceutical Sciences Review and Research. 2011;6(2) Article-014

4. J Sajan, Ta Cinu, Aj Chacko, J Litty and T Jaseeda, Tropical J Pharma Res, October 2009; 8 (5): 467-475.

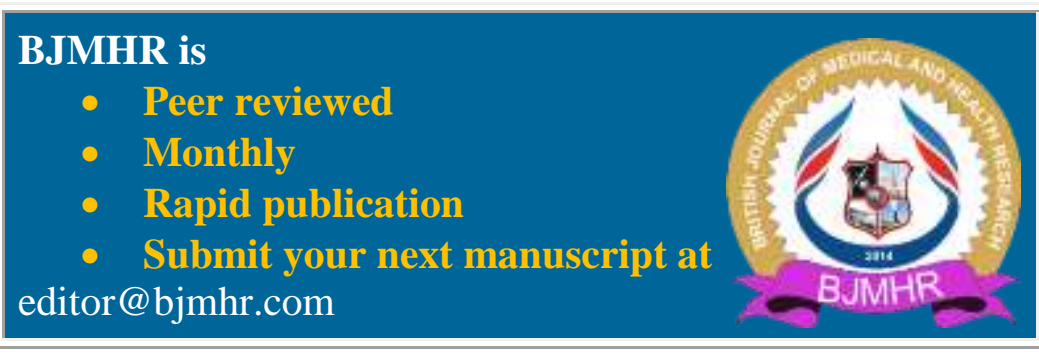

